\title{
émulations
}

\section{Pascal Martin - Les métamorphoses de l'assurance maladie. Conversion managériale et nouveau gouvernement des pauvres}

\section{Valentin Alibert}

Émulations - Revue de sciences sociales

2018, «Comptes rendus critiques, En ligne »

\section{Article disponible à l'adresse suivante}

https://ojs.uclouvain.be/index.php/emulations/article/view/7433

\section{Pour citer cet article}

Valentin Alibert, «Pascal Martin — Les métamorphoses de l'assurance maladie. Conversion managériale et nouveau gouvernement des pauvres », Émulations, en ligne. Mise en ligne le 1 er juillet 2018.

DOI : 10.14428/emulations.cr.043

Distribution électronique : Université catholique de Louvain (Belgique) : ojs.uclouvain.be

(C) Cet article est mis à disposition selon les termes de la Licence Creative Commons Attribution, Pas d'Utilisation Commerciale 4.0 International. http://creativecommons.org/licenses/by-nc/4.0/

Éditeur : Émulations - Revue de sciences sociales / Presses universitaires de Louvain https://ojs.uclouvain.be/index.php/emulations

ISSN électronique : 1784-5734

UCL PRESSES

UNIVERSITAIRES

DE LOUVAIN 


\title{
Pascal Martin - Les métamorphoses de l'assurance maladie. Conversion managé- riale et nouveau gouvernement des pauvres
}

\begin{abstract}
Valentin Alibert ${ }^{1}$
Recensé : Pascal Martin, Les métamorphoses de l'assurance maladie. Conversion managériale et nouveau gouvernement des pauvres, Rennes, Presses universitaires de Rennes, coll. « Le sens social», 2016, 243 p.
\end{abstract}

Cet ouvrage de Pascal Martin, tiré de sa thèse de doctorat soutenue en 2012, porte sur les conséquences de l'introduction de logiques managériales au sein de l'assurance maladie. L'auteur analyse ces bouleversements à l'aune des réformes néolibérales entreprises dans les années 1990 et s'inspirant du new public management (NPM) ${ }^{2}$. Il a ainsi étudié l'application de ces réformes au sein de l'assurance maladie et leur impact sur le travail de prise en charge des usagers, tout en démontrant « que la mise en œuvre de la CMU a engendré un nouveau gouvernement des pauvres qui institutionnalise une inégalité de traitement» (p. 233). Le livre de Martin analyse ces bouleversements en articulant « trois niveaux d'analyse » (p. 12) afin de mettre en évidence la manière dont une réforme impulsée de manière top-down se diffuse jusqu'aux agents de l'institution. Tout d'abord, une approche « macrosociologique » expose les orientations données par les politiques publiques à l'échelle étatique. Le deuxième niveau d'analyse s'inscrit à l'échelle de l'institution elle-même (la branche maladie de la Sécurité sociale), afin d'étudier la manière dont on met en œuvre concrètement ces orientations voulues par le gouvernement : d'après l'auteur, la nouvelle formation professionnelle des agents semble le levier d'action principal pour réformer l’institution. Enfin, l'analyse « microsociologique » met en évidence l'impact de ces réformes sur le travail d'accueil des usagers par les agents nouvellement formés aux techniques managériales.

Il convient tout d'abord d'inscrire ces réformes au sein d'un processus plus global de réduction du rôle de l'État dans un contexte de diminution des dépenses publiques. Ce travail de recherche permet ainsi d'explorer et de dévoiler un paradoxe : le renforcement du rôle de l’État au sein de la Sécurité sociale, pour en contrôler la gestion, semble

1 Étudiant en master de sciences sociales à l'ENS de Lyon.

${ }^{2}$ Comme le rappelle Colette Bec dans la préface, l'expression de new public management, utilisée depuis les années 1980, exprime la conversion de l'administration publique aux techniques de management issues du secteur privé. 
s'accompagner d'un effritement de «l'État social » (Castel, 1995). En effet, en interférant davantage, l'État s'inscrit en fait dans une tentative de désengagement. Par conséquent, dans le but d'effectuer des économies, l'assurance sociale entre dans un modèle concurrentiel, inspiré du NPM, avec la multiplication des assurances complémentaires. Or, ces réformes interviennent avec la mise en place d'un nouveau système d'assistance lié à la création de la couverture maladie universelle (CMU), l'objectif étant de « faire bénéficier d'une protection contre la maladie équivalente à celle de l'assurance maladie tous les individus français et étrangers vivant légalement sur le territoire et qui ne peuvent avoir droit à la Sécurité sociale » (p. 11). Elle est ensuite complétée par l'aide médicale de l'État (AME), destinée aux étrangers en situation irrégulière. Pourtant, face à l'universalisme affiché, les politiques publiques vont renforcer le contrôle d'accès à ces protections dans un souci de maîtrise des dépenses d'assistance.

Pour mener à bien cette recherche, Martin a effectué une enquête ethnographique reposant sur une observation participante des « deux côtés de la barrière » (p. 62) au sein d'une Caisse primaire particulièrement marquée par l'accueil de publics en grande précarité. L'auteur s'est tout d'abord placé dans la position de l'agent qui suit en interne la formation professionnelle destinée à recruter le personnel d'encadrement. Ainsi, après avoir été engagé en tant qu'agent de formation, le chercheur s'est retrouvé au plus près des enquêtés pour observer le travail de prise en charge des usagers. Cette deuxième observation participante pose néanmoins quelques problèmes lors de l'enquête. En effet, certains agents identifiant le chercheur à leur ancien formateur ou à un cadre de l'institution, le sociologue doit régulièrement effectuer un travail réflexif pour nuancer les propos de certains enquêtés influencés par cette situation d'interaction particulière. Cette double position s'avère néanmoins fructueuse dans sa recherche : en ayant lui-même suivi cette nouvelle formation, le chercheur peut ainsi analyser de manière précise limpact de la transmission des logiques managériales sur le travail des agents.

Les deux premiers chapitres permettent d'éclairer la construction de la protection sociale en France via une approche socio-historique. Le premier chapitre analyse la dichotomie entre une "logique d'assistance» (p. 27) individualisante, qui oppose «les bons » aux «mauvais pauvres », et une logique «assurancielle» portée par la mise en place de la Sécurité sociale en 1945, ayant pour objectif « la disparition de l'assistance sous sa forme moralisante originelle » (p. 27). L'auteur voit alors dans la mise en place de la CMU un retour vers la logique d'assistance, distinguant ses usagers et leur demandant de justifier leur situation. Le deuxième chapitre explore l'importance de la formation professionnelle permettant de passer de la logique de solidarité à celle d'efficacité, les nouveaux employés ayant à intérioriser « les schèmes de pensée » de la gestion managériale (p. 66). Les agents de direction sont ainsi formés comme des gestionnaires, les cadres intermédiaires intègrent le management et les agents subalternes doivent appréhender l'usager comme un client. Cette formation professionnelle s'accompagne 
d'une typologie des publics rattachés à un imaginaire moralisant qui entraîne une gestion et une prise en charge variable des usagers.

Le troisième chapitre est particulièrement dense : l'auteur y étudie la manière dont les réformes managériales et les nouvelles formations induisent des pratiques différenciées chez les agents. Tout d'abord, Martin démontre le renforcement des hiérarchies entre agents : « Le classement des usagers en deux groupes, selon qu'ils relèvent de l'assurance maladie ou de l'assistance, trouve son correspondant dans la division du travail au sein des équipes d'accueil » (p. 109). Ainsi, les employés les plus anciens traitent les assurés sociaux, tandis que les prétendants à la CMU et à l'AME, aux situations plus précaires, sont pris en charge par des emplois jeunes ou par des employés récemment formés aux techniques managériales. Par ailleurs, cette logique managériale impose aux agents que l'efficacité prenne le pas sur la solidarité, bouleversant ainsi la philosophie de leur travail. Le critère d'évaluation des agences devient désormais le temps d'attente des usagers : les agents reçoivent comme mot d'ordre l'orientation de ces derniers dans leurs démarches suite à un premier traitement au pré-accueil qui doit s'effectuer en moins de trois minutes. Cette injonction quantitative induit, d'une part, un contrôle accru des agents par leur hiérarchie et, d'autre part, un renforcement des tensions au travail. En effet, les agents de la Sécurité sociale formés avant ces réformes n’adhèrent pas à cette nouvelle logique : le risque mis en avant par l'auteur sur la base des entretiens menés avec ces agents est celui d'une perte de repères de certains employés qui ont l'impression de voir disparaître les valeurs qui justifiaient le sens de leur action. Enfin, l'analyse de la gestion différenciée des publics est particulièrement stimulante. En effet, cette gestion différenciée est à la fois spatiale et temporelle, dépendant de l'orientation décidée au pré-accueil de la Caisse primaire : les assurés sociaux sont immédiatement dirigés vers l'étape suivante pour un traitement plus rapide de leur dossier tandis que les demandeurs CMU sont renvoyés chez eux après une prise de rendezvous ultérieure. Même si la référence n'est pas mobilisée par l'auteur, cette inégale prise en charge s'inscrit au sein du « système des inégalités » mis en évidence par Alain Bihr et Roland Pfefferkorn : en effet, la recherche de Martin démontre bien la dimension « cumulati [ve] » des inégalités (Bihr, Pfefferkorn, 2015 [2008] : 55). Si les usagers les plus précaires éligibles à la CMU ou à l'AME disposent d'un "capital bureaucratique " (p.139) moindre (maîtrise de la langue, capacité à monter des dossiers...), ils doivent faire face à des demandes de justificatifs qui sont plus nombreuses en raison d'un contrôle accru dans le cadre d'une lutte contre la fraude renforcée. Par ailleurs, leur traitement est retardé par l'impératif de prise de rendez-vous. Enfin, ils sont aidés par des employés moins qualifiés (contrats jeunes) ou par des agents directement formés aux logiques managériales d'efficacité plutôt que de solidarité. Cette inégale prise en charge s'avère motivée par la " volonté de réduire le nombre de visiteurs à l'accueil physique, d'invisibiliser la file d'attente, d'exclure les corps des pauvres » (p. 121).

Enfin, les deux derniers chapitres s'attachent au processus de contrôle et d'exclusion à l'œuvre à travers ces évolutions de l'assurance maladie. L'auteur analyse tout d'abord 
la «politique de restriction progressive de l'État social» (p. 187) qu'il observe notamment du fait d'un durcissement des conditions d'attribution de la CMU aux ressortissants roumains et bulgares (chapitre 4). Puis, en intitulant le dernier chapitre « Le gouvernement des pauvres ", Martin révèle la position de dépendance des usagers les moins informés, soumis au bon vouloir des agents dans la constitution de leur dossier. L'agent dispose ainsi d'un « pouvoir discrétionnaire » (p. 202) : il peut se donner le droit de moraliser l'usager et même de le punir « pour le principe » (p. 221), en raison de la position de supériorité que lui confère l'institution. Par ce terme de " gouvernement », le sociologue pointe du doigt un paradoxe majeur dans l'évolution de l'assurance maladie : la solidarité comme philosophie de l'aide sociale s'accompagne désormais d'une fonction de contrôle, à l'image de la vérification renforcée des titres d'identité. L'auteur analyse cette ambivalence en reprenant largement les travaux de Pierre Bourdieu dont la citation suivante est mise en exergue par Colette Bec dès la préface de l'ouvrage de Martin : «les structures étatiques liées au welfare state [...] contrôlent d'autant mieux qu'elles servent» (Bourdieu, 2012 : 243).

On peut prolonger l'analyse de l'auteur concernant le désenchantement de certains agents, qui doutent du bien-fondé de leur action en raison d'un impératif quantitatif qui passe avant la qualité de la prise en charge, en étudiant la dimension émotionnelle de leur travail. L'ouvrage de Martin ne propose pas d'analyser explicitement la difficulté d'une gestion émotionnelle face à des récits d'usagers qui cherchent l'empathie des agents. Pourtant, cette gestion semble d'autant plus difficile que l'impératif d'accélération est une norme désormais imposée à tous les agents. En s'appuyant sur les travaux d'Arlie Russell Hochschild (2017), on pourrait alors y voir une difficulté supplémentaire en termes de "travail émotionnel». Selon Hochschild, ce travail, qui se multiplie depuis les années 1980 avec l'importance croissante du secteur des services, « requiert d'un individu qu'il déclenche ou refoule une émotion dans le but de maintenir extérieurement l'apparence attendue, apparence qui doit produire sur les autres l'état d'esprit adéquat » (Hochschild, 2017 : 27). Dans le cas de l'accueil des usagers, le travail ne nécessite pas de produire une émotion. Cependant, l'intériorisation de ces nouvelles normes amène à ne pas faire preuve d'empathie face à la détresse des usagers. «C'est un travail qui mentalement est extrêmement éprouvant. [...] tu es obligé de te protéger aussi, tu ne peux pas absorber toute la misère du monde » (p. 156) : l'extrait de cet entretien avec un agent révèle le travail de «redéfinition du moi » (Hochschild, 2017 : 151) nécessaire pour que l'individu ne se confonde pas avec un rôle professionnel d'autant plus éloigné de lui-même qu'il n'en partage pas les nouveaux principes d'action et qu'il a lui-même travaillé autrement, selon d'autres principes, auparavant.

En conclusion, la lecture de cet ouvrage s'effectue sans difficulté : Martin appuie régulièrement son propos sur des extraits d'entretiens et restitue avec précision les observations menées à l'échelle fine de la Caisse primaire. On apprécie tout particulièrement l'analyse du dispositif spatial de l'accueil. En effet, les nombreux détails donnés 
permettent de saisir la manière dont l'espace sert à contrôler les corps (discipline imposée par la file d'attente) et à dominer les usagers : la mise à distance spatiale d'une file d'attente le plus près possible de la sortie participant de la mise à distance sociale entre les usagers et les agents. En cela, ce travail de recherche pourra intéresser non seulement des sociologues, mais également des géographes sensibles aux dispositifs spatiaux de contrôle des individus à l'échelle micro-locale. Plus largement, l'ouvrage intéressera tous ceux qui s'interrogent sur les mutations de l'aide sociale dans un contexte économique de restriction budgétaire.

L'ouvrage de Martin s'avère du reste salutaire en raison de la contribution qu'il apporte à la critique d'un système déshumanisant et inadapté aux logiques de solidarité pourtant mises en avant entre 1945 et 1975, pendant la phase d'universalisation de la protection sociale. Son analyse déconstruit ainsi les stéréotypes de l'assistanat, de l'usager fraudeur et profiteur comme résurgence de la figure du « mauvais pauvre ». Il révèle également les préjugés racistes qui gravitent autour de la prise en charge des étrangers, parfois inconsciemment intériorisés chez certains agents qui usent et abusent de leur « pouvoir discrétionnaire » au nom d'une action moralisante. À ce titre, la formule proposée par Colette Bec dans la préface est tout à fait justifiée : en dévoilant des transformations en inadéquation avec le projet initial de la Sécurité sociale, Martin endosse un triple statut : celui « d'acteur [...], d'analyste, mais aussi [...] de citoyen » (p. 8).

\section{Bibliographie}

BiHR A., PfEFferkorn R. (2015 [2008]), Le système des inégalités, Paris, La Découverte («Repères. Sociologie »).

Bourdieu P. (2012), Sur l'État : cours au Collège de France (1989-1992), Paris, France, Seuil (« Raisons d'agir »).

CASTEL R. (1995), Les métamorphoses de la question sociale : une chronique du salariat, Paris, Fayard (« L’Espace du politique »).

HochsCHILD A. R. (2017), Le prix des sentiments : au cœur du travail émotionnel, Paris, France, La Découverte. 